\title{
Editorial
}

\section{Synthesis: the first step in chemistry!}

\author{
by M. I. Azócar \\ Facultad de Química y Biología, Universidad de Santiago de Chile, Santiago, Chile. \\ manuel.azocar@usach.cl
}

Every chemist has at some time synthesized a compound, because synthesizing is the beginning of all subsequent studies. It does not matter if the applications include medicine, engineering, food, energy, or it is just the interest in creating new molecules. Most discoveries in chemistry include the synthesis of new materials and every day new compounds are created or found, and innovative preparation routes are tested.

According to the Cambridge Structural Database (CSD), in 1993 only $38.9 \%$ of the records reported the presence of transition metal elements, with 20,439 structures. 17 year later that figure increased to $48.0 \%$, with 120,638 new structures, and recently, in 2012, a new record was reached, with 319,188 structures, representing $53.5 \%$ of the total in the CSD [1]. For that reason it is common, even in many prestigious inorganic chemistry journals, to find that in a large number of articles there is a section on synthesis and spectroscopic and/or structural characterization. The word "synthesis" appears in all papers and is one of the most common words in the titles of articles.

In the last decades inorganic synthesis has evolved, reaching a great variety of techniques as published in the book "Modern Inorganic Synthetic Chemistry", with more than 20 chapters on different aspects of such diverse areas as: high and low temperature synthesis, hydrothermal and solvothermal synthesis, coordination polymers, cluster compounds, host-guest materials, ceramics, nanomaterials, among other chapters devoted exclusively to modern inorganic synthesis [2].

But not only the traditional synthesis and preparation of inorganic compound is growing every day. Over the last ten years there has been an increasing number of new highly ordered structures called "nanoparticles," expanding even more the family of inorganic materials with a great impact all the way from medicine to energy. Consequently, this promising area of science shows more than 100 journals associated with new nanomaterials in the last 20

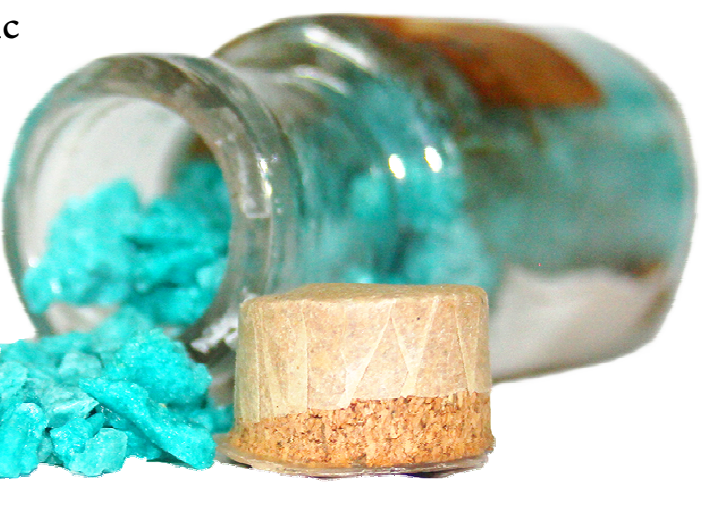




\section{Editorial}

years [3]. Therefore, synthesis actually has a central role in chemistry, physics and biology, with an extraordinary level of sophistication and unpredictable applications.

In the words of 2001 Chemistry Nobel Prize laureate Ryoji Noyory in his article "Synthesizing our future", Chemistry has a central role in science and synthesis has a central role in chemistry [4], and it is not strange to find the works of Nobel Prize in chemistry winners [5] directly associated with new molecules and their first step in chemistry: Synthesis.

\section{References}

[1] Cambridge Crystallography Database Center. http://www.ccdc.cam.ac.uk

[2] Xu, R.; Pang, W. and Huo, Q. Eds. Modern inorganic synthetic chemistry. Elsevier, 2011.

[3] Lohse, S.E.; Murphy, J.C. J. Am. Chem. Soc. 2012, 134, 15607-15620

[4] Noyori, R. Nature Chemistry, 2009, 1,5-6.

[5] http://www.nobelprize.org/nobel_prizes/chemistry/laureates/ 